\title{
CURRENT REVIEW
}

\section{Excitatory Dentate Granule Cells Normally Contain GAD and GABA, but Does That Make Them GABAergic, and Do Seizures Shift Granule Cell Function in the Inhibitory Direction?}

\author{
Robert S. Sloviter
}

Departments of Pharmacology and Neurology, University of Arizona College of Medicine, Tucson, Arizona

$\mathrm{F}$ ew subjects in the field of epilepsy research are more actively discussed than the possible defects and disturbances in the $\gamma$-aminobutyric acid (GABA) system that might cause epilepsy. The early finding that baby food deficient in vitamin $\mathrm{B}_{6}$ (a cofactor of the GABA-synthesizing enzyme GAD) caused vitamin-reversible seizures provided one of the first clues that seizures might be caused by reduced synthesis of GABA $(1,2)$. Accordingly, GABA neurons have been alternately proposed to be highly vulnerable or relatively invulnerable after insults known to cause epilepsy $(3,4)$, and GABAmediated inhibition is reportedly decreased, increased, lost and recovered, or none of these, in a variety of constantly compared but greatly dissimilar animal models (5). A number of recent articles focused on the seemingly paradoxic idea that the glutamatergic granule cells of the hippocampal dentate gyrus, which are possibly the most lethally excitatory cells in the brain, also may be GABAergic and may shift their functional state in the inhibitory direction after seizures occur (6). If GABA neurons are defined as cells normally expressing GAD and GABA, then granule cells are clearly GABA neurons, but what does that mean when everyone knows that granule cells are excitatory (7)? Before diving into the reductionist world of

Address correspondence to RS Sloviter, Department of Pharmacology, University of Arizona College of Medicine, 1501 N. Campbell Ave., Tucson, AZ 85724-5050; E-mail: sloviter@u.arizona.edu

Epilepsy Currents Vol. 3, No. 1 (January/February) 2003 pp. 3-5

Blackwell Science Inc.

(C) American Epilepsy Society experimental minutiae in which an endless number of possible mechanisms can be argued endlessly, and in which a broad perspective is the first casualty, it may be worthwhile to consider the "big picture."

In Eccles' (8) view, normal synaptic inhibition forms a barrier through which only strong excitatory signals can penetrate. The "sculpting away" or "filtering out" of weak excitatory signals by this tonically active inhibitory barrier focuses strong excitation to its intended target cells, sharpens the message, and suppresses unwanted responses of adjacent cells meant to be excluded from the conversation. Keeping excitatory signals temporally and spatially focused allows a relatively small structure to receive, integrate, and send out a large number of distinct messages meant for different destinations. When both weak and strong depolarizations produce action potentials, which is what occurs whenever $\mathrm{GABA}_{\mathrm{A}}$ receptors are blocked, seizure discharges result. Given this obvious connection between GABAergic failure and seizures, every conceivable disturbance in the process that begins with the formation of GABA neurons during development and culminates in the reuptake of GABA released from presynaptic terminals has been suggested to be a cause of epilepsy.

One of the clearest parts of the otherwise unsolved puzzle of human epilepsy is the role that the granule cells of the hippocampal dentate gyrus play in producing the pattern of hippocampal cell loss called hippocampal sclerosis. Dentate granule cells survive most insults that cause epilepsy, and they have giant presynaptic terminals filled with glutamate, which when released, kills most of the cells targeted by these powerful excitatory neurons $(9,10)$. Our thinking about granule cells and their possible role in epilepsy has evolved considerably during the last 30 years. In 1973, Crawford and Connor (11) concluded that the clearly excitatory mossy fiber pathway used glutamate as its transmitter, but this report was largely ignored because glutamate was assumed at the time to be just a simple amino acid. Strangely, simplicity often garners little respect. Nonetheless, the subsequent identification of glutamate receptors and the development of specific glutamate-receptor antagonists eventually led to the acceptance that glutamate is our main excitatory transmitter, and that the mossy fibers are glutamatergic. The more recent realization that, like many neurons, granule cells contain several additional neuroactive substances, including zinc and dynorphin, has not disturbed the view that granule cells are fundamentally glutamatergic and excitatory, because we think of fast-acting small amino acid transmitters in one category and most other neuroactive 
substances as "neuromodulators" of the actions of the primary transmitter.

In 1991, Sandler and Smith (12) reported that ultrastructurally identified mossy fiber terminals could bind GABA antibody molecules, but their suggestion that mossy fibers may normally contain functional GABA was largely ignored because the GABA-synthesizing enzyme GAD was "known" to be absent from the mossy fibers. This suggested that the small amount of GABA-like immunoreactivity detected in individual mossy fiber terminals was the result of either GABA uptake from the extracellular fluid, or simply nonspecific binding of GABA antibody molecules. As it turns out, there are two forms of GAD, GAD65 and GAD67, and the mossy fibers do not normally contain detectable GAD65 (13). However, by using a newly available antiserum specific for GAD67 and improved methods for detecting GABA, we discovered that dentate granule cells of rats and monkeys express GAD67 and GABA in abundance in the normal state (6). The constitutive expression of both GABA and its synthetic enzyme in otherwise glutamatergic neurons clearly indicated that granule cells normally produce and contain two small-molecule, fast-acting neurotransmitters, one excitatory and one inhibitory. My colleagues and I also observed that experimental seizures increased granule cell GAD65 and GAD67 messenger RNA (mRNA), as well as both GAD proteins, and increased dentate gyrus GABA concentrations sixfold. Thus we inferred that seizures might shift the functional phenotype of dentate granule cells in the inhibitory direction (6).

So why do normal granule cells contain GAD67 and GABA in abundance? Does granule cell GABA play a transmitter role normally? And why do seizures rapidly increase GAD and GABA synthesis selectively in granule cells, but not in hippocampal pyramidal cells? Perhaps granule cells need to produce and use GABA because they are so intrinsically powerful and potentially lethal to their normal target cells. Clearly, finding GAD and GABA in granule cells does not prove a transmitter role for mossy fiber GABA. However, the evidence is growing. Although the presence of vesicular GABA transporter protein in granule cells has not yet been demonstrated, Lamas et al. (14) now provide evidence for mossy fiber vesicular GABA transporter mRNA, and the data from Gutierrez $(15,16)$ and Walker and colleagues (17) provide evidence that mossy fiber GABA is released and produces postsynaptic effects that can be antagonized by $\mathrm{GABA}_{\mathrm{A}}$-receptor blockade. Assuming that all of the criteria are eventually met for concluding that GABA is normally and abnormally an inhibitory transmitter at mossy fiber synapses, what might that mean? Well, that all depends on one's conception of how mossy fibers normally do what they do.

Studies by Weisskopf et al. (18) and Vogt and Nicoll (19) on the functional roles of mossy fiber dynorphin and GABA are consistent with Eccles' view that targeted cells need to receive highly focused excitation, with adjacent neuronal elements needing to be inhibited. Lateral inhibition is a wellaccepted principle in the cerebellum (8), but it has received relatively little attention in the hippocampus (20), which shares a similar highly laminar structural organization. In the cerebellum, the excitatory parallel fibers of cerebellar granule cells target a thin slice of Purkinje cells, which are the cerebellar output cells. At the same time, inhibitory interneurons whose axons inhibit Purkinje cells in adjacent "lamellae" also are excited within the target zone, thereby producing "lateral" inhibition. Because the focused excitatory input to a slice of target cells strongly excites the center and weakly excites the outer edges of the target, lateral inhibition serves to suppress the weak activation at the edges and produce a more coherent central excitation (8). This functional organization of the cerebellum, if operational in the hippocampus, might explain why granule cells form a highly lamellar projection to their target cells, and why inhibitory interneurons are such prominent targets of the mossy fiber pathway (21). As a conceptual framework for future studies, perhaps it would be useful to think of the mossy fiber pathway as so powerful and so highly focused on exciting its thin slice of target cells that it requires a variety of inhibitory substances that, when released, produce both pre- and postsynaptic effects that focus glutamate-mediated excitation to their intended targets and inhibit collateral players $(18,19)$.

In this context, the observation that granule cell GAD and GABA are rapidly increased after granule cell seizure discharges and kindling $(6,22)$ may reflect a compensatory mechanism that counteracts abnormal hyperactivity. It seems reasonable to deduce that seizures may represent a violation of a constantly and carefully monitored upper limit on "normal" excitation, which causes a functional shift in the inhibitory direction through increased synthesis of mossy fiber GAD and GABA (6). We have hypothesized that neuronal loss after epileptogenic injuries may break down lateral inhibitory barriers, allowing abnormally large aggregates of neurons to fire synchronously and overcome inhibition entirely $(10,20)$. If so, the breakdown in lateral inhibitory barriers may trigger a compensatory increase in GABA synthesis that limits the lateral effects of mossy fiber glutamate release and postsynaptic excitation.

One hypothetical role for mossy fiber GABA that has not been considered previously relates to the literature suggesting that after injury, synaptic reorganization forms recurrent connections between normally unconnected granule cells $(23,24)$. If the formation of aberrant granule cell-granule cell connections also involves a change of mossy fiber phenotype in the inhibitory direction, then mossy fiber sprouting may constitute the formation of recurrent inhibitory, rather than excitatory connections, which would be consistent with the granule 
cell hyperinhibition observed in vivo after kainate-induced status epilepticus (25). Although no clear experimental data yet indicate that increased mossy fiber GAD and GABA shift the phenotype of granule cells in the inhibitory direction, it seems highly unlikely that granule cell GAD and GABA are conserved from mouse to human and are rapidly increased by abnormal excitation, for no useful purpose. Undoubtedly, time will tell.

\section{References}

1. Hunt AD, Stokes J, McCrory WW, Stroud H. Pyridoxine dependency: report of a case of intractable convulsions in an infant controlled by pyridoxine. Pediatrics 1954;13:140-145.

2. Bankier A, Turner M, Hopkins IJ. Pyridoxine-dependent seizures-a wider clinical spectrum. Arch Dis Child 1983;58:415-418.

3. Ribak CE, Harris AB, Vaughn JE, Roberts E. GABAergic nerve terminals decrease at sites of focal epilepsy. Science 1979;205:211-214.

4. Sloviter RS. Decreased hippocampal inhibition and a selective loss of interneurons in experimental epilepsy. Science 1987;235:73-76.

5. Dalby NO, Mody I. The process of epileptogenesis: a pathophysiological approach. Curr Opin Neurol 2001;14:187-192.

6. Sloviter RS, Dichter MA, Rachinsky TL, Dean E, Goodman JH, Sollas AL, Martin DL. Basal expression and induction of glutamate decarboxylase and GABA in excitatory granule cells of the rat and monkey hippocampal dentate gyrus. J Comp Neurol 1996;373:593-618.

7. Yamamoto C. Activation of hippocampal neurons by mossy fiber stimulation in thin brain sections in vitro. Exp Brain Res 1973;14: 423-435.

8. Eccles JC. The understanding of the brain. New York: McGraw-Hill, 1973.

9. Sloviter RS. Permanently altered hippocampal structure, excitability, and inhibition after experimental status epilepticus in the rat: the dormant basket cell hypothesis and its possible relevance to temporal lobe epilepsy. Hippocampus 1991;1:41-66.

10. Sloviter RS. The functional organization of the hippocampal dentate gyrus and its relevance to the pathogenesis of temporal lobe epilepsy. Ann Neurol 1994;35:640-654.

11. Crawford IL, Connor JD. Localization and release of glutamic acid in relation to the hippocampal mossy fiber pathway. Nature 1973; 244:442-443.
12. Sandler R, Smith AD. Coexistence of GABA and glutamate in mossy fiber terminals of the primate hippocampus: an ultrastructural study. J Comp Neurol 1991;303:177-192.

13. Ribak CE, Vaughn JE, Saito K. Immunocytochemical localization of glutamic acid decarboxylase in neuronal somata following colchicine inhibition of axonal transport. Brain Res 1978;140:315-332.

14. Lamas M, Gomez-Lira G, Gutierrez R. Vesicular GABA transporter mRNA expression in the dentate gyrus and in mossy fiber synaptosomes. Mol Brain Res 2001;93:209-214.

15. Ramirez M, Gutierrez R. Activity-dependent expression of GAD67 in the granule cells of the rat hippocampus. Brain Res 2001;917: 139-146.

16. Gutierrez R. Seizures induce simultaneous GABAergic and glutamatergic transmission in the dentate gyrus-CA3 system. J Neurophysiol 2000;84:3088-3090.

17. Walker MC, Ruiz A, Kullmann DM. Monosynaptic GABAergic signaling from dentate to $\mathrm{CA} 3$ with a pharmacological and physiological profile typical of mossy fiber synapses. Neuron 2001;29:703-715.

18. Weisskopf MG, Zalutsky RA, Nicoll RA. The opioid peptide dynorphin mediates heterosynaptic depression of hippocampal mossy fibre synapses and modulates long-term potentiation. Nature 1993;362: 423-428.

19. Vogt KE, Nicoll RA. Glutamate and gamma-aminobutyric acid mediate a heterosynaptic depression at mossy fiber synapses in the hippocampus. Proc Natl Acad Sci U S A 1999;96:1118-1122.

20. Sloviter RS, Brisman JL. Lateral inhibition and granule cell synchrony in the rat hippocampal dentate gyrus. J Neurosci 1995;15: 811-820.

21. Acsady L, Kamondi A, Sik A, Freund T, Buzsaki G. GABAergic cells are the major postsynaptic targets of mossy fibers in the rat hippocampus. J Neurosci 1998;18:3386-3403.

22. Schwarzer C, Sperk G. Hippocampal granule cells express glutamic acid decarboxylase- 67 after limbic seizures in the rat. Neuroscience 1995;69:705-709.

23. Tauck D, Nadler J. Evidence of functional mossy fiber sprouting in hippocampal formation of kainic acid-treated rats. J Neurosci 1985; 5:1016-1022.

24. Sutula T, Xiao-Xian H, Cavazos J, Scott G. Synaptic reorganization in the hippocampus induced by abnormal functional activity. Science 1988;239:1147-1150.

25. Sloviter RS. Possible functional consequences of synaptic reorganization in the dentate gyrus of kainate-treated rats. Neurosci Lett 1992; 137:91-96. 